\title{
Mouse Genetics: ONKO CHISHIN -study the old, to understand the new-
}

\author{
Michihiko Sugimoto \\ Technology and Development Team for Mammalian Genome Dynamics, RIKEN BioResource Center, \\ 3-1-1 Koyadai, Tsukuba, Ibaraki 305-0074, Japan
}

Following the rediscovery of Mendel's laws, research fields based on genetics began to grow dramatically. Simultaneously, the development of inbred strains of mice started in the early 20th century, since when they have become widely used as model animals not only in genetics but also, for example, in medical science, developmental biology, cell biology, epigenetics and genome biology. The expansion of utilization and standardization of inbred strains has made mouse genetics one of the most important research fields in genetics. Therefore, it is no exaggeration to say that mouse genetics plays a leading role in the advancement of life sciences research. In this issue of Genes and Genetic Systems, two review papers on mouse genetics by members of the Genetics Society of Japan are provided.

In the first paper, Oka and Shiroishi review studies about genetic incompatibility in mammals as revealed by mouse genetics. Hybrid sterility, which may be caused by genetic incompatibilities between subspecies/species, is a representative phenomenon of postzygotic reproductive isolation, leading to speciation during evolution. Genetic tests have shown that elements associated with hybrid sterility are disproportionately located on the $\mathrm{X}$ chromosome. Oka and Shiroishi discuss the impact of the divergence of $\mathrm{X}$-linked genes on hybrid sterility, and propose models for genetic incompatibility arising from variants in cis-trans regulatory factors.

In the second paper, I introduce $t$-haplotype studies.
The $t$-haplotype is a variant form of the proximal onethird of mouse chromosome 17 . Numerous mutations, including a class of recessive lethal mutations known as ' $t$-complex lethal mutations', reside within this recombination-suppressed region. To better understand mammalian development, many geneticists had tried to pinpoint the genes responsible for these lethal mutations for more than 80 years since the discovery of the $t$-haplotype, and one of them was finally identified in 2012 for the first time. I summarize $t$-haplotype research, focusing particularly on mouse development and including the results of molecular identification of this first $t$-complex lethal gene.

Diverse research fields in mammalian biology are now based on mouse genetics, because the homogeneity of the genetic background in each inbred mouse strain is useful for simplifying and understanding complex traits in mammals, especially to elucidate gene interactions and gene dosage effects. Abundant mutant mouse resources are also very important tools for understanding mammalian development and gene function. I therefore believe that mouse genetics will become ever more important for life sciences research.

Dr. Kazuo Moriwaki, one of the pioneers of mouse genetics, passed away on November 23, 2013. I sincerely dedicate this special review issue to the memory of Dr. Moriwaki with deep thanks for his great contribution to mouse genetics. 